\title{
Properties of co-deposited layers on graphite high heat flux components at the TEXTOR tokamak
}

\author{
E. Fortuna ${ }^{\text {a,* }}$, M.J. Rubel ${ }^{\text {b }}$, V. Philipps ${ }^{\text {c }}$, K.J. Kurzydłowski ${ }^{\text {a }}$, Ph. Mertens ${ }^{\text {c }}$, \\ M. Miśkiewicz ${ }^{\text {a }}$, M. Pisarek ${ }^{\text {a }}$, G. Van Oost ${ }^{\mathrm{d}}$, W. Zieliński ${ }^{\text {a }}$ \\ ${ }^{a}$ Faculty of Materials Science and Engineering, Warsaw University of Technology, EURATOM-IPPLM, PL-02-507 Warsaw, Poland \\ ${ }^{\mathrm{b}}$ Alfvén Laboratory, Royal Institute of Technology, Association EURATOM-VR, S-100 44 Stockholm, Sweden \\ ${ }^{\mathrm{c}}$ Institute of Plasma Physics, Forschungszentrum Jülich, Association EURATOM-FZJ, D-52425 Jülich, Germany \\ d Department of Applied Physics, Ghent University, Rozier 44, B-9000 Ghent, Belgium
}

\begin{abstract}
The objective of this work was to examine the structure, composition and properties of co-deposited films from the TEXTOR tokamak. Hydrogenated films formed on the toroidal belt pump limiter (ALT-II), and on the ICRF antenna grill were studied using a set of material analysis techniques. Plasma edge diagnostics were used to assess the parameters influencing the film formation during discharges auxiliary heated by ICRF. The essential results are summarized as follows: (i) the distribution of plasma impurities co-deposited in the films is non-uniform and (ii) the surface topography, crystallographic structure, fuel retention and composition (i.e., content of re-deposited plasma impurities) of the films show significant diversity depending on the location where they were formed. These differences are associated with the local geometry, the tokamak operation scenarios and the resulting plasma edge properties.
\end{abstract}

(c) 2007 Elsevier B.V. All rights reserved.

\section{Introduction}

High heat flux components in most present-day tokamaks are made of graphite or/and carbon fibre composites. The major drawback of carbon is its reactivity with hydrogen isotopes leading to large material erosion, migration and, eventually, to a significant fuel content in co-deposited layers [1-3]. This, in turn, is the major reason for careful studies of the layers in carbon-wall machines, because castellated carbon components are to be used in ITER

\footnotetext{
* Corresponding author. Tel.: +48 2223487 48; fax: +48 22 2348750.

E-mail address: elaf@inmat.pw.edu.pl (E. Fortuna).
}

in the strike-point zone. This will ensure good power handling but one may also expect significant carbon erosion followed by migration and re-deposition of carbonaceous species leading eventually to a high fuel inventory. On plasma exposed areas, the deposited carbon films are also subject to high heat fluxes, particularly in high power plasma scenarios or special plasma configurations. Additional metallic impurities originating from erosion of nongraphite parts of the wall will be co-deposited simultaneously with carbon, thus forming mixed carbon-metal layers. This may influence both mechanical stability of the films and fuel retention. Therefore, various aspects of co-deposition should be studied in detail. 


\section{Experimental}

The study was carried out for two types of codeposited layers formed in the TEXTOR tokamak: (i) films formed during $\sim 14000 \mathrm{~s}$ of plasma operation on the graphite plates of the toroidal belt pump limiter (ALT-II) and (ii) co-deposited layers formed during $\sim 25 \mathrm{~h}$ of tokamak operation on the stainless steel grill of the rf antenna. ALT -II (Advanced Limiter Test II) is the main limiter defining the minor radius $(a=46 \mathrm{~cm})$, whereas two arrays of antennas for ion cyclotron resonance frequency (ICRF) heating are located at the radius of $50 \mathrm{~cm}$.

The topography and basic structure of the films were recorded by scanning electron microscopy (SEM). The information on the internal structure of these materials was obtained via scanning transmission electron microscope (STEM), whereas their elemental composition was determined using X-ray photo-electron spectroscopy (XPS) and electron probe micro-analysis (EPMA) with energy and wavelength dispersive spectrometers (EDS and WDS, respectively).

Plasma edge diagnostic techniques such as the sniffer probe [4], surface collector probe [5] and laser-induced fluorescence [6] were applied to determine the density, energy and fluxes of species during ohmically and ICRF heated (1 MW) discharges in TEXTOR. The goal was to assess the influence of heating mode on the production of impurity species (i.e., the composition of the scrape-off layer, SOL) and, consequently, the influence on the co-deposit formation. Targets exposed to the SOL with the surface collector probe and co-deposited layers from various locations in the tokamak were examined ex situ by means of Rutherford backscattering spectroscopy (RBS) and nuclear reaction analysis (NRA).

\section{Results}

The SEM images in Fig. 1(a) and (b) show the micro-structure of co-deposited layers peeled off the ALT II plate and the antenna grill, respectively. The formation of strata is observed in both cases, but the fine features are quite different. The film from the limiter plate is fairly smooth and uniform as revealed by TEM studies performed on samples from several positions. The smallest observed elements of the nanostructure have a size of tenths of nanometres and they are aligned in chains. In most cases the diffraction patterns indicate a complete
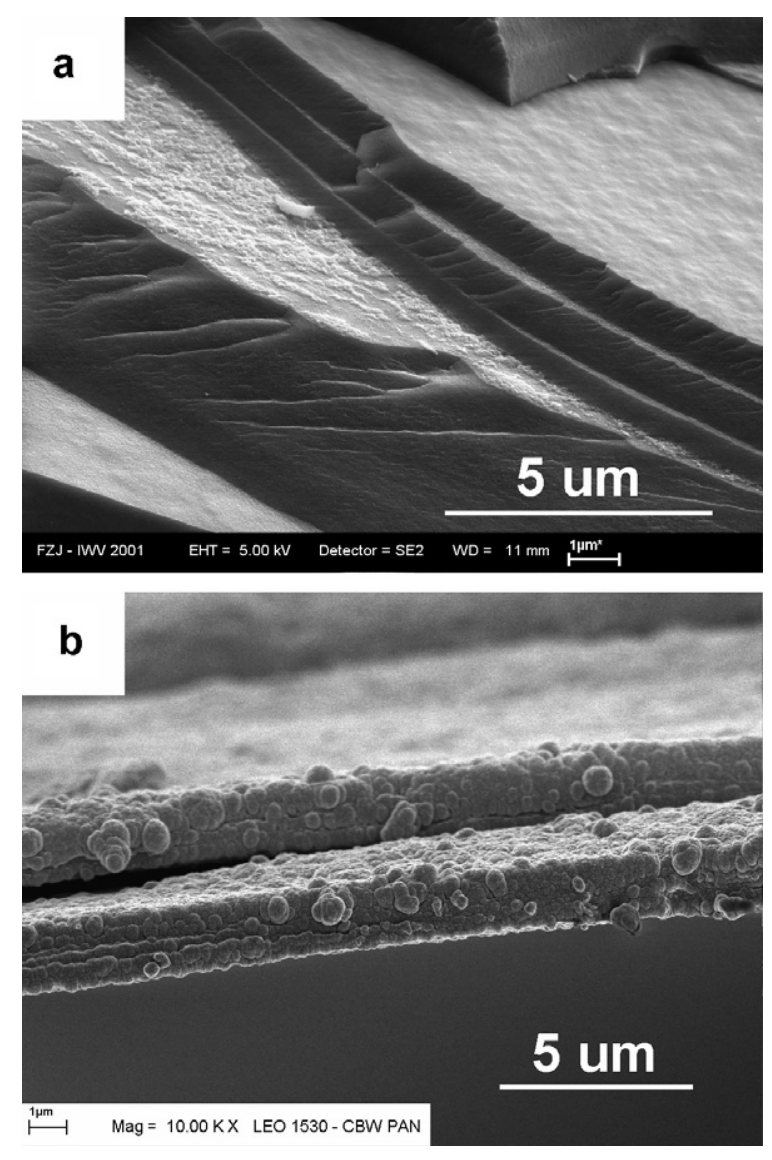

Fig. 1. Co-deposited layers formed on the ALT-II graphite tile (a) and the antenna (b).

lack of a crystallographic arrangement of the lattice, confirming the amorphous nature of such films. In some areas, the sharpening of the diffraction rings might indicate a certain order of the amorphous matter. EDS analyses associated with SEM revealed a non-uniform distribution of the elements in this type of deposit. Mapping of several elements shown in Fig. 2 clearly proves the presence of microregions enriched with either carbon, silicon, or chromium (the distributions of $\mathrm{Cr}$ and $\mathrm{Fe}$ were the same). These results confirm previous TEM and EELS (electron energy loss spectroscopy) studies by Muto [7], and SIMS (secondary ion mass spectrometry) analysis by Hildebrandt [8] showing an inhomogeneous distribution of carbon and boron in such films. The various elements in the deposit (Fig. 2) have quite different origins. Carbon is eroded and re-deposited from the poloidal and toroidal graphite limiters. Silicon originates from wall protective siliconised coatings, whereas the presence of iron (and other metallic species: $\mathrm{Cr}$ and $\mathrm{Ni}$ ) is associated 


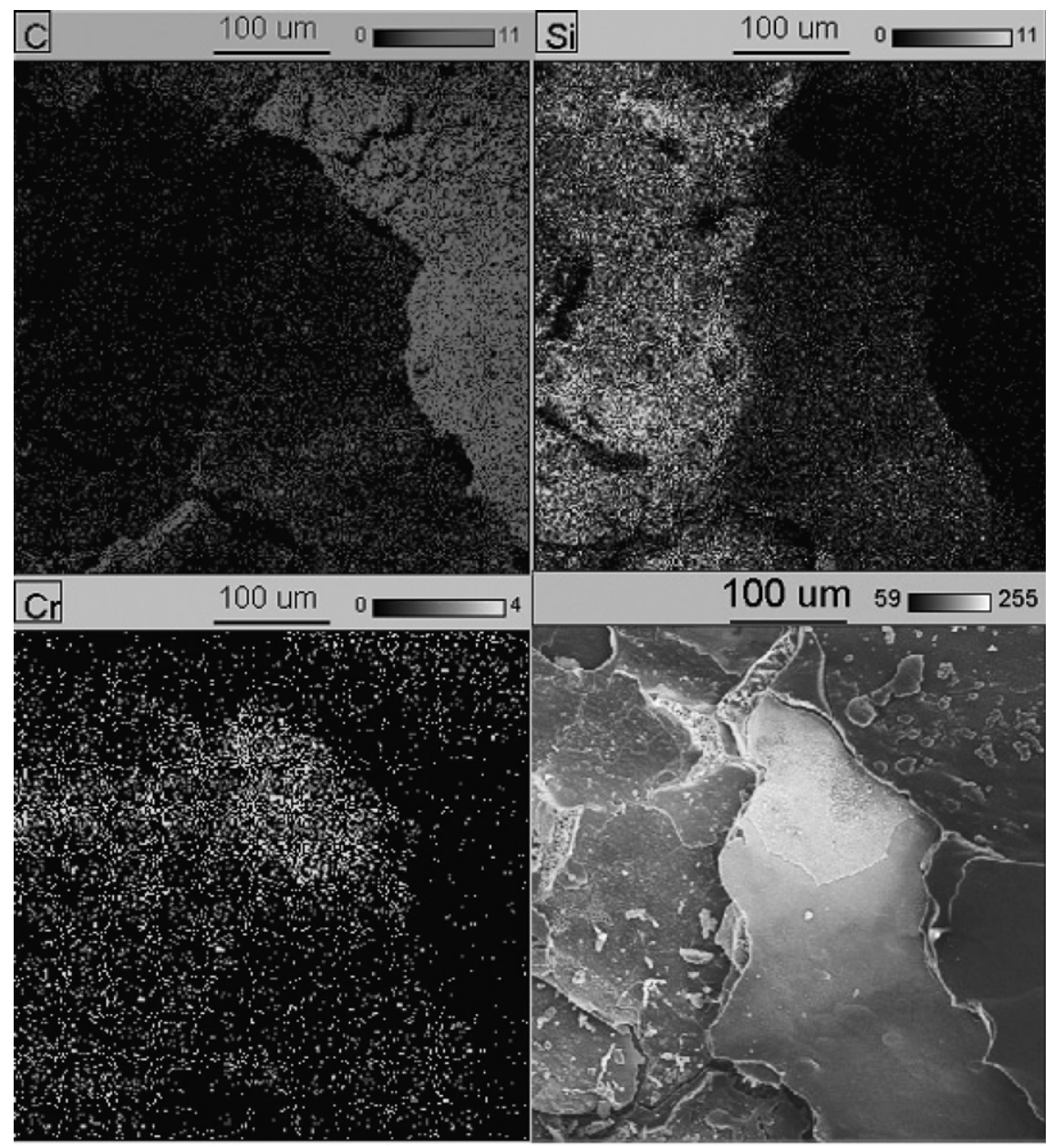

Fig. 2. SEM picture of the deposit from the graphite tile indicating areas with increasing concentration of carbon, silicon and chromium.

with the erosion of the Inconel ${ }^{\circledR}$ liner. The deuterium content determined with a ${ }^{3} \mathrm{He}^{+}$based NRA in these films is in the range $9-12$ at.\%, as reported in detail elsewhere [5]. Apart from carbon and deuterium, the most abundant element in the layers is boron which results from frequent boronisations of the entire inner wall of TEXTOR.

The material deposited on the ICRF antenna grill (Fig. 1(b)) appears as an agglomerate of fine granules with the smallest particle size of $30 \mathrm{~nm}$. It is similar to the deposit found on graphite tiles from the protection screen of that antenna [9]. The STEM image and diffraction pattern in Fig. 3 present characteristic micro-features of the deposit removed from the grill. The structure is very inhomogeneous and diversified. The agglomerates (indicated by arrows on the image) are surrounded by amorphous

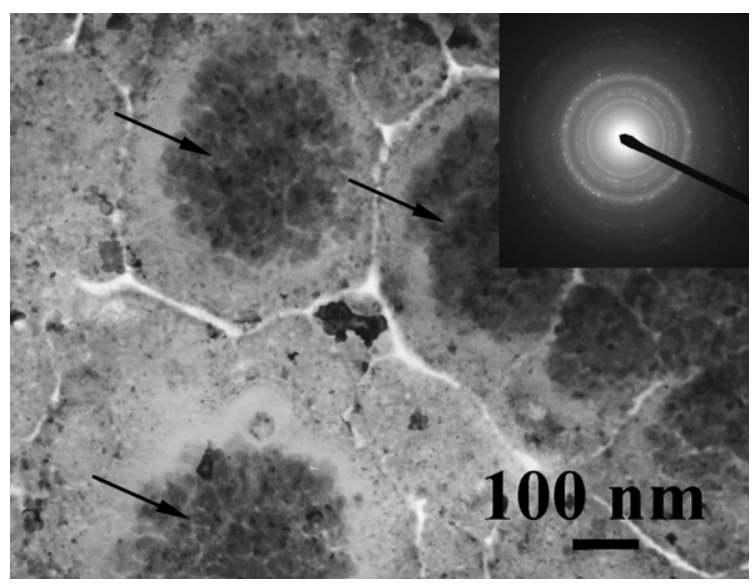

Fig. 3. TEM image together with the corresponding diffraction pattern for the deposit from the antenna grill. Arrows indicate areas with agglomerates. 
Table 1

Elemental composition (atomic \%) of the examined co-deposited layers

\begin{tabular}{lll}
\hline Element & ALT-II & Antenna \\
\hline $\mathrm{C}$ & $75-80$ & $24-27$ \\
$\mathrm{O}$ & $10-15$ & $15-17$ \\
$\mathrm{Fe}$ & $\sim 1$ & $20-23$ \\
$\mathrm{Si}$ & $1-10$ & $23-28$ \\
$\mathrm{~B}$ & $3-10$ & - \\
$\mathrm{Cr}$ & - & $6-7$ \\
\hline
\end{tabular}

matter. Internal surfaces and some voids are also identified. The diffraction pattern reveals both crystalline and amorphous material. As determined by EDS analysis during STEM studies, the main elements in the regions with a large number of crystallites are $\mathrm{Si}, \mathrm{C}$ and $\mathrm{Fe}$. Some quantities of $\mathrm{Ni}, \mathrm{Cr}$ and Mo are also detected. The deuterium concentration determined with NRA on the antenna protection screens was below 5 at.\% [9], i.e., at least a factor of two lower than on the ALT-II plates.

Elemental analyses were performed by means of XPS after $20 \mathrm{~min}$ of etching with $\mathrm{Ar}^{+}$ions (raster size: $6 \mathrm{~mm} \times 6 \mathrm{~mm}$ ). The results (in units of at.\%) for the plasma impurity species found in the two types of co-deposits are compiled in Table 1. The main component of the flakes from the ALT-II tile is carbon together with other elements such as B (3-10\%), O (15-20\%), Si (up to 10\%) and metallic elements ( $\mathrm{Fe}, \mathrm{Ni}, \mathrm{Cr}$ and $\mathrm{Mo}$ ) with a fraction of typically below $1 \%$. The scatter in the results is associated with the varying concentrations from placeto-place due to the non-uniform distribution and changes in composition, from layer-to-layer, as demonstrated in our earlier studies [10]. This particularly refers to the diverse content of B and Si that were used for the wall conditioning. On the antenna grill, the carbon content is only $25-30 \%$. This amount is comparable to the concentrations of heavier species such as $\mathrm{Fe}$ and $\mathrm{Si}$. The high content of iron can be attributed to the local erosion and redeposition of this element on the stainless steel grill. The oxygen detected in both deposits partly originates from the direct co-deposition with other plasma impurities, and partly from the ad- and absorption of oxygen-containing molecules during the exposure of co-deposits to air [11].

\section{Discussion and concluding remarks}

The overall morphology of the two types of codeposited layers differs significantly. The most remarkable differences are in the structure and the content of carbon and deuterium. The reason for this diversity is most probably due to conditions accompanying the film formation: the temperature, angle of particle impact and the energy range of incoming species. This also points clearly to the importance and influence of the plasma edge and SOL modification by the ICRF pulses which change the plasma-wall interactions by increasing the edge temperature and altering the electric sheath and ion trajectories [12].

The collector probe operated either in the timeresolved or time-integrated mode (experimental details can be found in [5]) was used to measure and compare the ion fluxes in the SOL under various heating conditions: ohmic, ICRF and neutral beams. The time-resolved measurements performed show an increase of deuterium and metal impurity fluxes by $20-30 \%$ in comparison to the ohmic phase of the discharge [11]. The results of the time-integrated measurements (i.e., exposure during the entire pulse) performed under ohmic and ICRF conditions in the siliconised machine are compiled in Table 2. The ICR auxiliary heating (two antennas - AN1 and AN2 - were in use) enhances the release of impurities. The effect is particularly significant for metals, indicating that they are released not only from the vessel walls (liner) but - probably predominantly - directly from the antennas. This is associated with: (i) the production of fast ions which can directly hit the antenna screen in the SOL of the outboard wall and (ii) a general increase of the ion impact energies on the antenna due to the sheath rectification effects. Several measurements done under various heating modes (e.g., with the sniffer probe; technical details in [4]) show that the energy of hydrogenic ions that exceed the sheath potential is $60-100 \mathrm{eV}$ in ohmic discharges and from 150 to $500 \mathrm{eV}$ during the ICRF pulses. One may assume

\section{Table 2}

Deposition rates of $\mathrm{B}, \mathrm{Si}$ and metals $(\mathrm{Me}=\mathrm{Ni}+\mathrm{Fe}+\mathrm{Cr})$ on the collector probe exposed to ohmically $(\Omega)$ and ICRF heated discharges

\begin{tabular}{lllc}
\hline Heating & $\begin{array}{l}\mathrm{Si} \\
\left(10^{15} \mathrm{~cm}^{-2} \mathrm{~s}^{-1}\right)\end{array}$ & $\begin{array}{l}\mathrm{B} \\
\left(10^{14} \mathrm{~cm}^{-2} \mathrm{~s}^{-1}\right)\end{array}$ & $\begin{array}{l}\mathrm{Me} \\
\left(10^{13} \mathrm{~cm}^{-2} \mathrm{~s}^{-1}\right)\end{array}$ \\
\hline$\Omega$ & 1.8 & 3.4 & 5.0 \\
ICRF & 5.1 & 4.6 & 12.8 \\
$\quad(\mathrm{AN}-1)$ & & & \\
$\Omega(\mathrm{A})$ & 4.3 & 1.1 & 8.8 \\
ICRF & 5.1 & 6.9 & 13.0 \\
$\quad(\mathrm{AN}-2)$ & & &
\end{tabular}

$\mathrm{AN}-1$ and $\mathrm{AN}-2$ denote the antenna sets in use. 
that the energy of other ion species is also significantly enhanced.

ICRF also increases the density of atomic deuterium in the SOL by a factor of 1.5 to 3 , as proven by laser-induced fluorescence at Lyman- $\alpha$. The pulse does not change the velocity distribution $(<2 \mathrm{eV})$. This is attributed to the presence of high energy ions which induce the release of hydrogen accumulated in the walls. The re-erosion rate of carbon deposited on the grill may then be increased, especially at high surface temperatures. As a consequence, carbon would be preferentially removed from co-deposited layer on the antenna, due to chemical erosion caused by hydrogenic species and physical sputtering. The co-deposited layer is then enriched with heavier elements (metals and $\mathrm{Si}$ ) that are more difficult to sputter and do not form volatile compounds in chemical processes. Such effects are also seen in the inner divertor of JET, where the deposits are strongly enriched with Be impurities [13].

The crystalline matter detected in such films may contain compounds (e.g., SiC) formed due to high temperatures and the high-energy ion impact. The difference in the structure of both films (flat laminar on ALT-II and granular on the grill) also points to the importance of the ion impact angle. The interaction at the toroidal limiter occurs at a very shallow angle of $1^{\circ}-2^{\circ}$, whereas the ion impact angle on the antenna is probably larger (distorted electric sheath and increased ion energies leading to effectively larger Larmour orbits) allowing the granular or columnar structures to be formed.

The present study has highlighted some conditions influencing the morphology of co-deposited layers. It has been shown that fuel may be retained in films containing relatively small amounts of carbon. This could have important consequences when developing techniques for fuel and co-deposit removal in fusion devices because layers enriched with metals are also expected in ITER. Therefore, such films as those identified on the antenna grill may be useful for developing and testing methods of deposit disintegration and removal for the nextstep machine where the use of carbon will be reduced.

\section{Acknowledgements}

This work was partly carried out under Contract of Association EURATOM-IPPLM and partly funded by EURATOM and, the Swedish Research Council (VR) under the Contract VR-621-20033170 .

\section{References}

[1] V. Philipps et al., Vacuum 67 (2002) 399

[2] J.P. Coad, M. Rubel, C.H. Wu, J. Nucl. Mater 241-243 (1997) 408.

[3] C.H. Skinner et al., J. Nucl. Mater 241-243 (1997) 214.

[4] V. Philipps et al., J. Nucl. Mater 162-164 (1989) 550.

[5] M. Rubel, P. Wienhold, in: K.H. Spatschek, J. Uhlenbusch (Eds.), Akademie Verlag, Berlin, 1994, Chapter 5.8.

[6] Ph. Mertens, P. Bogen, Appl. Phys. A43 (1987) 197.

[7] S. Muto et al., J. Nucl. Mater 307-311 (2002) 1289.

[8] D. Hildebrandt et al., Phys. Scr. T81 (1999) 25.

[9] M. Rubel et al., J. Nucl. Mater 266-269 (1999) 1185.

[10] P. Wienhold et al., J. Nucl. Mater. 313-316 (2003) 311.

[11] M. Rubel et al., J. Nucl. Mater 161 (1989) 153.

[12] J.M. Noterdaeme, G. Van Oost, Plasma Phys. Control. Fus. 35 (1993) 1481.

[13] J.P. Coad et al., J. Nucl. Mater 290-293 (2001) 224. 